\title{
The lung torsion dilemma: Detorsion without resection or resection without detorsion?
}

\author{
Pascal A. Thomas, MD, FETCS
}

\author{
From the Department of Thoracic Surgery, North University Hospital, Aix-Marseille University, Marseille, France. \\ Disclosures: Author has nothing to disclose with regard to commercial support. \\ Received for publication April 17, 2016; accepted for publication April 18, 2016; available ahead of print May 12, \\ 2016. \\ Address for reprints: Pascal A. Thomas, MD, FETCS, Department of Thoracic Surgery, North University Hospi- \\ tal, Aix-Marseille University, France (E-mail: pathomas@ap-hm.fr). \\ J Thorac Cardiovasc Surg 2016;152:746 \\ 0022-5223/\$36.00 \\ Copyright (C) 2016 by The American Association for Thoracic Surgery \\ http://dx.doi.org/10.1016/j.jtcvs.2016.04.063
}

Pulmonary torsion is a rare but probably underdiagnosed and underreported life-threatening event, especially in postoperative settings. Dai et $\mathrm{al}^{1}$ should be congratulated on having gone through with a fine-tooth comb near twothirds of a century's worth of medical literature on the topic. For sure, one could argue, as the authors themselves did, that the material of their review must be weakened by the long study period, insufficient clinical details, and especially by a strong publication bias related to the preferential report of atypical clinical presentations and favorable outcomes. However, they succeeded in extracting out of their comprehensive analysis some useful key messages regarding disease characteristics, distribution, and prognostic factors to enlighten how to prevent and treat this potentially disastrous condition.

Lung torsion has been mainly reported after lobectomy or lung transplantation, and only secondarily in trauma settings or following huge pleural effusion and various invasive chest procedures. It consists of an axial, most often clockwise, rotation of the lung or lobar bronchovascular pedicle with resultant airway and vascular obstructions. Its pathophysiology is not well understood, especially for those very rare so-called spontaneous torsions. A major predisposing factor seems to be an inadequacy of volume between the enclosing and the contents; that is, a small lung in a big chest cavity. In addition, this review ${ }^{1}$ clearly shows that even if any individual lobe or lung may be involved, the right middle lobe is typically endangered after upper lobectomy, specifically if the oblique fissure is complete, and should be fixed to the lower lobe during surgery.

The onset of sepsis should not be awaited to evoke this complication, the diagnosis of which remains based above all on chest radiography findings. Conclusive investigations are bronchoscopy showing the typical "fish mouth" bronchial twisting, and infused chest computed tomography scan showing vessel occlusion or tapering at the site of the pedicle torsion. Without prompt recognition and treatment, it evolves toward parenchymal ischemia, infarction,

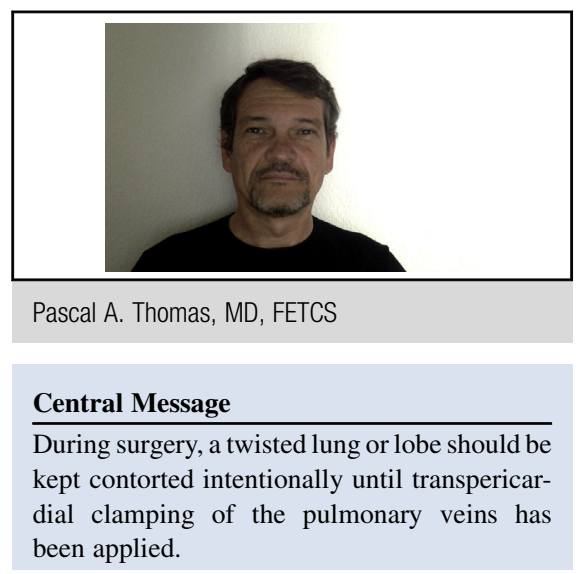

See Article page 737.

See Editorial page 658.

and finally fatal gangrene, especially if the whole lung is involved. The most valuable information coming from this review relates to treatment. Indeed, as soon as the diagnosis is highly suspected, early surgical exploration is mandated. Detorsion without resection can be safely performed only within the very first few hours of the event. Beyond this point, because of irreversible ischemic damage, resection without detorsion would prevent the risk of massive inflow of inflammatory mediators into the systemic circulation, which could trigger a systemic inflammatory response and potentially result in fatal multiorgan failure. Clot migration released from the obstructed pulmonary vein may also occur and cause a fatal stroke. For clinical practice, it can be deduced that the lung or lobe should be kept contorted intentionally until transpericardial clamping of the pulmonary veins has been applied. Then, the lobe or lung can be repositioned and afterward surgical decisions can be made whether resection should be performed or may be avoided.

The authors are to be commended for their great work that undoubtedly represents a helpful contribution to our knowledge on lung torsion.

\section{Reference}

1. Dai J, Xie D, Wang H, He W, Zhou Y, Hernández-Arenas LA, et al. Gening Jiang, MD Predictors of survival in lung torsion: A systematic review and pooled analysis. J Thorac Cardiovasc Surg. 2016;152:737-45.e3. 